\title{
WISE: A Web-Based Intelligent Sensor Explorer Framework for Publishing, Browsing, and Analyzing Sensor Data over the Internet
}

\author{
Kien A. Hua, Rui Peng, and Georgiana L. Hamza-Lup \\ School of Computer Science, University of Central Florida \\ Orlando, FL 32816, U.S.A \\ \{kienhua, rpeng, ghamza\}@cs.ucf.edu
}

\begin{abstract}
In this paper, we present WISE, an Internet-scale framework for publishing, browsing, and analyzing real-time sensor data. This environment enables providers to advertise their sensors on the Internet, and unsolicited users to locate desired sensors through a scalable peer-to-peer search facility. A specialized browser allows a user to remotely control the incoming data streams which activate corresponding plug-ins, such as a visualization module, to present the data in an intelligible way. Therefore, WISE also facilitates sharing of sensor data analysis tools in the form of software plug-ins.
\end{abstract}

\section{Introduction}

Nowadays pervasive sensing systems ranging from short-range wireless ad hoc sensor networks to more powerful capture devices are becoming a reality. As more and more sensor-based services become part of our daily life, they call for new technologies that enable publishing, searching, and browsing sensor data on the Internet. Consider the following scenario. A stream monitoring sensor network is deployed in a brook by a group of geographers. The sensors measure water temperatures, turbidity and precipitation, and periodically send back the data to an Internet-connected computer. These data arrive as a stream and are published on the Internet by the geographers. Independently, a biologist, studying the life zone in the same brook, searches for the information on the Internet. Within a few seconds, she learns about the data published by the geographers, and starts to browse and analyze the data stream on her own computer. As another example, a government might want to stimulate economy by publishing real-time traffic data, collected through sensing devices along expressways. Availability of such information would encourage development of many new commercial products such as a more intelligent GPS system that can reroute a trip when recognizing slow traffic ahead. In order to enable such kind of services on sensor data, we propose in this paper a new framework, called WISE (Web-based Intelligent Sensor Explorer), for sensor information sharing over the Internet. We envision an all-new Internet where innumerable sensing systems, deployed by numerous publishers, exposing different data which can be shared freely 
with a wide variety of unsolicited users, much like the way web pages are publicly shared today.

Recently there has been a growing interest on sensor data management with research activities focusing mainly on either: (1) dealing with packet routing and power conservation issues in sensor networks [3], or (2) managing the sensor networks at the application layer as a distributed database [2, 5, 7]. These techniques view the entire sensor network logically as a distributed database sharing the same schema. These schemes, though effective in their defined problem scales (i.e., tailored to supporting a given data provider to serve a predefined group of users), will not work well in the new sensor data sharing environment that we envision in this paper. The proposed WISE framework is NOT a sensor database management system. We summarize some of the differences between the current state-of-the-art and the proposed environment in Table 1.

Table 1. Differences between Sensor Database Management Systems and WISE

\begin{tabular}{|c|c|c|}
\hline & $\begin{array}{c}\text { Sensor Database } \\
\text { Management Systems }\end{array}$ & Proposed WISE \\
\hline Number of Data Providers & One & Numerous \\
\hline Data Users & Targeted users & Unsolicited users \\
\hline Schema Design & Homogeneous & Heterogeneous \\
\hline Data Usage & $\begin{array}{c}\text { to support specific } \\
\text { applications }\end{array}$ & $\begin{array}{c}\text { Ad hoc browsing of data } \\
\text { sources through a browser }\end{array}$ \\
\hline
\end{tabular}

\section{WISE Framework}

We revisit the scenario of the geographers to illustrate how this framework facilitates this application. To publish the data, a Sensor Data Description Advertisement (SDDA) is created on a Sensor Data Server (SDS) to hold the meta-data, and publish them into the Internet. At the same time, the sensor proxy [4] collects intermittent data from sensors in the brook, packages incoming sensor data into tuples and forwards them to the SDS. When a biologist looking for related sensors enters search criteria into a local Sensor Data Browser (SDB), this software uses a distributed search facility to look for relevant SDDAs. As the browser displays a list of relevant SDDAs on the screen, the biologist identifies the desired sensor and clicks on it. In response, the browser establishes a session with the SDS using a Sensor Stream Control Protocol (SSCP), gets the real-time stream using a Sensor Stream Transport Protocol (SSTP), and directs the data to the Visualizer for display. The various components are described in the following subsections.

\subsection{Canonical SDDA Format}

Each SDDA is an XML document for advertising a sensor service consisting of a collection of related sensors. A sample SDDA is given in Fig. 1. 


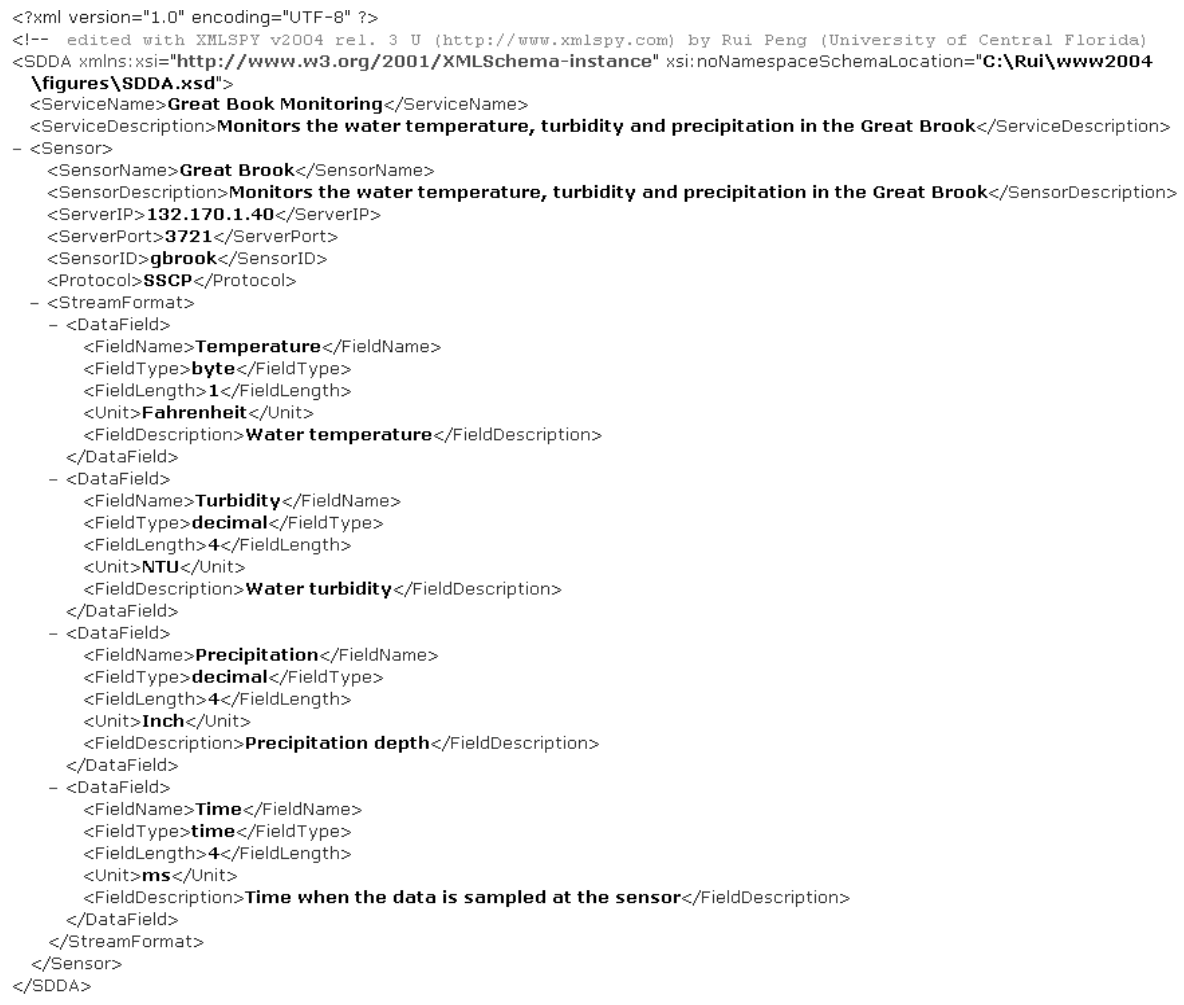

Fig. 1. A sample SDDA

\subsection{Sensor Data Server (SDS) and Sensor Data Browser (SDB)}

The SDS is responsible for managing incoming data from sensor proxies and delivering the data to SDBs. It has three major components, namely the Publish Manager, the Stream Manager, and the Session Manager. The Publish Manager accepts registrations from sensor proxies and publishes the corresponding SDDAs into the Internet. The Stream Manager manages buffers for incoming data, and directs them to either local applications, or remote SDBs, or both. The Server Session Manager accepts client requests and control messages using SSCP and delivers the stream to clients using SSTP. A client uses two separate channels to interact with the server, one for control messages, and one for data. The control channel is bidirectional, but the data channel only goes in one direction.

The SDB consists of two components, the Client Session Manager and a simple Visualizer module. The Client Session Manager has three major functionalities. First, it is responsible for accepting user search criteria and discovering SDDAs on the Internet. Second, it connects to SDS's and establishes a client session for each incoming stream. A client session also has two channels, control channel and data 
channel. Third, it directs data to the Visualizer module for presentation to the user. The Visualizer is responsible for displaying the incoming data stream on the screen.

\subsection{Communication Protocols}

Two application-layer communication protocols are used in WISE framework, namely SSCP and SSTP. The SSCP is used for control message exchange on the control channel between an SDS and an SDB. As an analogy, the SSCP acts as a network remote control providing a set of useful methods for a client to control the "playback" of the sensor stream. SSCP does not deliver the stream itself, but rather uses SSTP for real-time data transmission. The control methods are as follows.

- PREPARE. Request the SDS to allocate resources for a sensor stream and prepare for the data delivery.

- START. Request the SDS to start the data transmission through the data channel. This operation can also be used to resume data transmission after a pause operation.

- PAUSE. Temporarily stop the stream transmission without releasing server resources. Any data transmitted during the pause period are lost.

- STOP. Notify the SDS to stop the data transmission and free server resources.

Before sending a data frame, the SDS wraps it with an SSTP header. The sequence number in the header increments by one for each SSTP packet and is used by the SDB to detect packet loss and ensure in-order delivery.

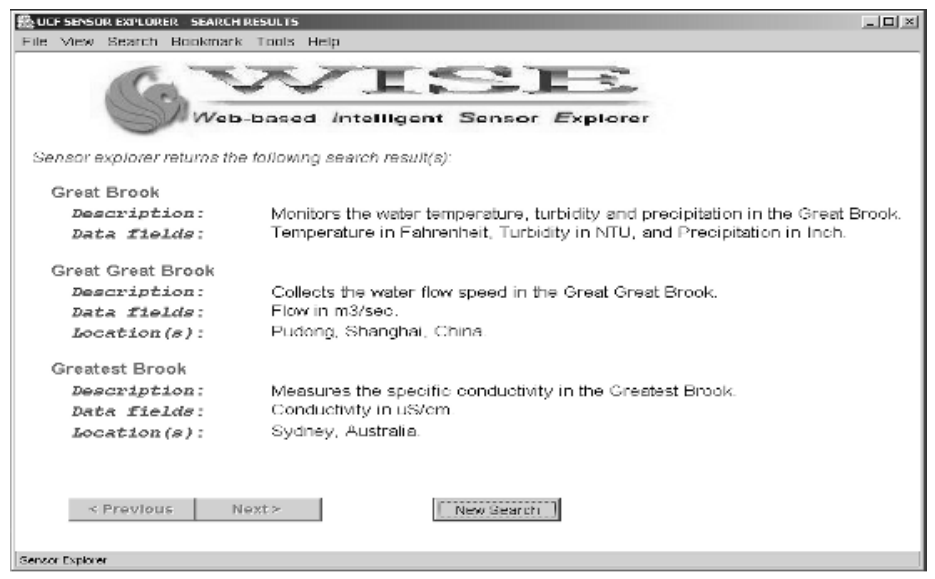

Fig. 2. The WISE sensor browser

\subsection{Search Mechanism}

Although WISE can be configured to work with any search engine as long as it recognizes the sensor metadata, we opt for a peer-to-peer search mechanism to avoid bottleneck and achieve better scalability. Peers in WISE use a distributed hash table 
(DHT) function to facilitate index storage and access. When an SDS publishes an SDDA, it is cached by a nearby service peer, and its index is pushed to the network according to the hash function. When an SDB searches for an SDDA, the SDB issues a query to a nearby service peer, which calculates the DHT function and finds the peer that contains the index for the SDDA. The SDB then gets the index from that peer and finds the target SDDA. Interested readers are referred to [6] for a detailed discussion of this search technique.

\section{Prototype}

We have developed a WISE prototype using the design and techniques presented in the last section. The current version has a simple user interface with a basic set of functionalities. A screen shot of the SDB is shown in Fig. 2. The user can enter a set of keywords as the search criteria. After the results are brought up by the browser, the user can select a service by clicking on its name. This will activate the Visualizer which allows the user to choose from a number of different visualization methods.

The current prototype is intended as a proof of concept. Future research will provide a more complete browser with functionalities such as the plug-in capability. We also need to do a more in-depth evaluation of the effectiveness and scalability of the search mechanism.

\section{References}

1. E. H. Callaway, Wireless Sensor Networks: Architecture and Protocols, Auerbach Publications, 2003.

2. A. Deshpande, S. Nath, P. B. Gibbons and S. Seshan, "Cache-and-Query for Wide Area Sensor Databases," in SIGMOD, 2003.

3. D. Ganesan, D. Estrin, and J. Heidemann, "Dimensions: Why do we need a new data handling architecture for sensor networks?" in HotNets-1, 2002.

4. S. Madden and M. J. Franklin, "Fjording the Stream: An Architecture for Queries over Streaming Sensor Data," in ICDE, 2002.

5. S. Madden, M. J. Franklin, J. M. Hellerstein, and W. Hong, "TAG: A Tiny AGgregation Service for Ad-Hoc Sensor Networks," in OSDI, 2002.

6. Project JXTA, http://www.jxta.org.

7. Y. Yang and J. Gehrke, "Query Processing for Sensor Networks," in CIDR, 2003. 